\title{
European Resuscitation Council Guidelines for Resuscitation 2010
}

\section{European Resuscitation Council (ERC) Executive Committee}

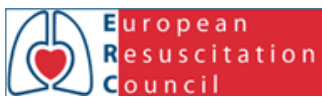

Chairman: Bernd W. Böttiger

Chairman and Director of the Department of Anaesthesiology and Postoperative Intensive Care Medicine, University of Cologne, Germany

Immediate Past Chairman: David Zideman

Consultant Anaesthetist and Hon Senior Lecturer,

Imperial College Healthcare NHS Trust, London, UK

Executive Director: Leo L. Bossaert

Emeritus Professor of Medicine, Cardiology and Intensive Care, University of Antwerp, Belgium

Hon. Treasurer: Jan Bahr

Center for Anesthetics, Emergency and Intensive Care Medicine, University Hospital Göttingen, Germany

Hon. Secretary: Koenraad G. Monsieurs

Consultant in Emergency Medicine, Emergency Department, Ghent University Hospital, Ghent, Belgium

ILCOR Liaison Officer: Jerry P. Nolan

Consultant in Anaesthesia and Intensive Care Medicine,

Royal United Hospital, Bath, UK

\section{ERC Guidelines Writing Group}

Gamal Abbas, Chief of Emergency Department, Al Rahba Hospital, Abu Dhabi, United Arab Emirates

Annette Alfonzo, Consultant Nephrologist, Queen Margaret Hospital, Dunfermline, Fife, UK

Hans-Richard Arntz, Senior physician,

Head of Emergency Medicine, Department of Cardiology Campus Benjamin Franklin Charite, Berlin, Germany

John H.W. Balance, Orchid Bank, Woolhope, Herefordshire, UK

Alessandro Barelli, Professor of Anaesthesia, Intensive Care Medicine and Clinical Toxicology, Catholic University School of Medicine, Rome, Italy

Michael A. Baubin, Associate Professor of Anaesthesiology,

Department of Anaesthesiology and Critical Care Medicine, University Hospital Innsbruck, Austria

Dominique Biarent, Associate Professor of Paediatrics, Paediatric Intensive Care and Emergency Medicine, Université Libre de Bruxelles, Queen Fabiola Children's University Hospital, Brussels, Belgium

Joost J.L.M. Bierens, Anesthesiologist, Maxima Medical Centre, Eindhoven, The Netherlands

Robert Bingham, Consultant Paediatric Anaesthetist, Great Ormond Street Hospital for Children London, UK
Leo L. Bossaert, Emeritus Professor of Medicine, Cardiology and Intensive Care, University of Antwerp, Antwerp, Belgium

Hermann Brugger, Associate Professor, Head of EURAC Institute of Mountain Emergency Medicine, Bozen, Italy

Antonio Caballero, Consultant in Emergency Medicine, Hospital Universitario Virgen del Rocío, Sevilla, Spain

Pascal Cassan, National Medical Advisor,

European Reference Centre for First Aid Education, French Red Cross, Paris, France

Maaret Castrén, Professor of Emergency Medicine, Department of Clinical Science and Education, Karolinska Institute, Stockholm, Sweden

Cristina Granja, Director of the Emergency and Intensive Medicine Department, Hospital Pedro Hispano, Matosinhos, Portugal, Affiliate Professor of Biostatistics and Medical Informatics, Faculty of Medicine, Porto, Portugal

Nicolas Danchin, Professor of Cardiology, Head,

Department of Coronary Artery Disease and Intensive Cardiac Care, Hôpital Européen Georges Pompidou, Paris, France

Charles D. Deakin, Consultant in Cardiac Anaesthesia and Critical Care, Southampton University Hospital NHS Trust, Southampton, UK

Joel Dunning, Registrar in Cardiothoracic Surgery,

Department of Cardiothoracic Surgery, James Cook, University Hospital, Middlesbrough, UK

Christoph Eich, Consultant Paediatric Anaesthetist,

Zentrum Anaesthesiologie, Rettungs- und Intensivmedizin Universitätsmedizin Göttingen, Germany

Marios Georgiou, Resuscitation Officer, Nicosia General Hospital, Nicosia, Cyprus

Robert Greif, Professor, Dept. Anesthesiology and Pain Therapy, Director Peripheral Anesthesia Division, Director Medical Education Program, University Hospital Bern, Inselspital, Bern, Switzerland

Anthony J. Handley, Honorary Consultant Cardiologist, Colchester Hospital University NHS Foundation Trust, Colchester, UK

Rudolph W. Koster, Department of Cardiology, Academic Medical Center, Amsterdam, The Netherlands

Freddy K. Lippert, Medical Director of Emergency Medicine and Emergency Medical Services, Head Office,

The Capital Region of Denmark Copenhagen, Denmark

Andrew S. Lockey, Consultant in Emergency Medicine, Calderdale and Huddersfield NHS Trust, Salterhebble, Halifax, UK

David J. Lockey, Consultant in Anaesthesia and Intensive Care Medicine, Frenchay Hospital, Bristol, UK

Jesús López-Herce, Pediatric Intensive Care Department, Hospital General Universitario Gregorio Marañón, Professor of Paediatrics, Complutense University of Madrid, Spain

Ian K. Maconochie, Consultant in Paediatric

Emergency Medicine \& Hon Senior Lecturer, St Mary's Hospital, Imperial College Healthcare NHS Trust, London, UK 
Koenraad G. Monsieurs, Consultant in Emergency Medicine, Emergency Department, Ghent University Hospital, Ghent, Belgium Nikolaos I. Nikolaou, Head of CCU, Constantopouleio General Hospital, Athens, Greece

Jerry P. Nolan, Consultant in Anaesthesia and Intensive Care Medicine, Royal United Hospital, Bath, UK

Peter Paal, Associate Professor, Department of Anesthesiology and Critical Care Medicine, University Hospital Innsbruck, Austria

Gavin D. Perkins, Associate Clinical Professor of Critical Care and Resuscitation, University of Warwick, Warwick Medical School, Warwick, UK

Violetta Raffay, Head of Emergency Medicine Mentors, Municipal Institute for Emergency Medicine Novi Sad, Novi Sad, AP Vojvodina, Serbia

Thomas Rajka, Consultant Paediatric Intensivist,

Oslo University Hospital, Kirkeveien Oslo, Norway

Sam Richmond, Consultant neonatologist,

Sunderland Royal Hospital, Sunderland, UK

Charlotte Ringsted, Professor and Director of Centre for Clinical

Education, University of Copenhagen and Capital Region, Denmark, Rigshospitalet, Copenhagen, Denmark

Antonio Rodríguez-Núñez, Associate Prof. of Paediatrics, University of Santiago de Compostela FEAS, Pediatric Emergency and Critical
Care Division, Pediatric Area Hospital Clinico Universitario de Santiago de Compostela, 15706 Santiago de Compostela, Spain

Claudio Sandroni, Assistant Professor of Anaesthesiology and Intensive Care, Catholic University School of Medicine, Policlinico Universitario Agostino Gemelli, Rome, Italy

Gary B. Smith, Critical Care and Resuscitation, University of Warwick, Warwick Medical School, Warwick, UK

Jasmeet Soar, Consultant in Anaesthesia and Intensive Care Medicine, Southmead Hospital, North Bristol NHS Trust, Bristol, UK

Petter Andreas Steen, Professor of Emergency Medicine, University of Oslo, Norway

Kjetil Sunde, Professor, Surgical Intensive Care Unit, Oslo University Hospital Ulleval, Oslo, Norway

Karl-Christian Thies, Consultant Anaesthetist,

Birmingham Children's Hospital, Birmingham, UK

Jonathan Wyllie, Consultant in Neonatology and Paediatrics, Clinical Director of Neonatology, The James Cook University Hospital, Middlesbrough, UK

David Zideman, Consultant Anaesthetist and Hon Senior Lecturer, Imperial College Healthcare NHS Trust, London, UK

\section{ERC-Leitlinien zur Kardiopulmonalen Reanimation 2010}

\section{Autorisierte deutschsprachige Übersetzung durch GRC, ARC und SRC}

\section{Verantwortlich für \\ die deutschsprachige Übersetzung:}

Dr. rer. nat. Dr. med. Burkhard Dirks, Vorsitzender des GRC Univ.-Doz. Dr. Michael Baubin, Vorsitzender des ARC

\section{German Resuscitation Council (GRC) -Vorstand}

Deutscher
Rat für
Wiederbelebung

Vorsitzender: Dr. rer. nat. Dr. med. Burkhard Dirks Sektion Notfallmedizin, Klinik für Anästhesiologie, Universitätsklinikum Ulm

Stellvertretender Vorsitzender: Prof. Dr. med. Hans-Richard Arntz Medizinische Klinik II, Klinikum Benjamin Franklin, Berlin

Schriftführer: Prof. Dr. med. Uwe Kreimeier

Klinik für Anaesthesiologie, Klinikum der Universität München

Schatzmeister: Stefan Osche

DRK-Generalsekretariat, Team 23, Berlin

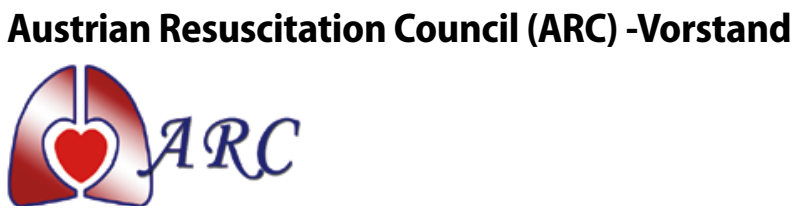

Vorsitzender: Univ.-Doz. Dr. Michael Baubin Universitätsklinik für Anästhesie und Intensivmedizin, Universitätsklinik Innsbruck

Stellvertretende Vorsitzende: Ass. Prof. Dr. Gudrun Burda Univ. Klinik f. Kinder- und Jugendheilkunde, Universitätsklinik-AKH Wien als Vertreterin der medizinischen Fachgesellschaften

Univ.-Prof. Dr. Hans Domanovits, Wien, als Vertreter für die medizinischen Universitäten

Dr. Karl Holaubeck, St. Pölten, als Vertreter für die Rettungsorganisationen

Präs. Dr. Michael Lang, Eisenstadt, für die Österreichische Ärztekammer, den Österreichischen Gesundheits- und Krankenpflege Verband, das österr. Bundesheer, den österr. Herzfonds Dr. Sabine Schneider-Klimanek und Dr. Rainer Schmid, Wien, kooptiert für die Arbeitsgruppe Innerklinische Reanimation Dr. Michael Hüpfl, Wien, kooptiert für die Arbeitsgruppe Trauma Univ.-Prof. Dr. Wolfgang Schreiber, Wien, kooptiert für das Österreichische Rote Kreuz 


\section{Swiss Resuscitation Council (SRC) -Vorstand}

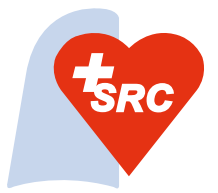

Präsident: Dr. med. Urs Klemmer

Schweizerische Rettungsflugwacht (REGA), Zürich

Past President: Dr. med. Martin Brüesch

UniversitätsSpital Zürich, Institut für Anästhesiologie, Zürich

Kassier: Jann Rehli

Vereinigung Rettungssanitäter Schweiz (VRS), Amriswil

\section{Übersetzer im Auftrag des GRC, ARC und SRC}

Prof. Dr. med. Hans-Richard Arntz, Medizinische Klinik II, Klinikum Benjamin Franklin, Charité Berlin, Hindenburgdamm 30, 12200, Berlin, E-Mail: hans-richard.arntz@charite.de

Dr. rer. pol. Jan Bahr, Zentrum Anaesthesiologie, Rettungs- und Intensivmedizin, Universitätsmedizin Göttingen, Robert-Koch-Straße 40, 37075 Göttingen,

E-Mail:jan.bahr@med.uni-goettingen.de

Univ.-Doz. Dr. Michael Baubin, Universitätsklinik für Anästhesie und Intensivmedizin, Anichstraße 35, A-6020, Innsbruck,

E-Mail: michael.baubin@uki.at

Dr. med. Stefan Braunecker,

Klinik für Anästhesiologie und Operative Intensivmedizin, Universitätsklinikum Köln, Kerpener Straße 62, 50937 Köln, E-Mail: stefan.braunecker@uk-koeln.de

Ass. Prof. Dr. med. Gudrun Burda, Univ. Klinik f. Kinder und Jugendheilkunde, Klinische Abt. f. Allg. Pädiatrie und Neonatologie, Währinger Gürtel 18-20, A-1090, Wien, E-Mail: gudrun.burda@meduniwien.ac.at

Dr. rer. nat. Dr. med. Burkhard Dirks, Sektion Notfallmedizin, Universitätsklinikum Ulm, Prittwitzstr. 43, 89075 Ulm, E-Mail: burkhard.dirks@uni-ulm.de
PD Dr. med. Christoph Eich, Zentrum Anaesthesiologie, Rettungs- und Intensivmedizin, Universitätsmedizin Göttingen, Robert-Koch-Str. 40, 37075 Göttingen, E-Mail: ceich@t-online.de

Prof. Dr. med. Hendrik W. Gervais, Klinik für Anästhesiologie Johannes Gutenberg-Universität, Langenbeckstrasse 1, 55131 Mainz, E-Mail: gervais@anaesthesie.klinik.uni-mainz.de

Peter Goldschmidt, Arbeiter-Samariter-Bund Deutschland e.V., Sülzburgstr. 140, 50937 Köln, E-Mail: p.goldschmidt@asb.de

Dr. med. Jan-Thorsten Gräsner, Universitätsklinikum SchleswigHolstein, Schwanenweg 21, 24105 Kiel, E-Mail:graesner@anaesthesie.uni-kiel.de

Prof. Dr. med. Robert Greif, Universitätshospital Bern, Inselspital, CH-3010 Bern, E-Mail: Robert.Greif@insel.ch

Dr. med. Urs Klemmer, Schweizerische Rettungsflugwacht (REGA), Postfach 1414, CH-8058 Zürich, E-Mail: Urs.Klemmer@rega.ch

Prof. Dr. med. Uwe Kreimeier, Universitätsklinikum München, Nussbaumstr. 20, 80376 München,

E-Mail: Uwe.Kreimeier@med.uni-muenchen.de

Dr. med. Ulrich Kreth, Kinderkrankenhaus St. Nikolaus, AKH Viersen GmbH, Hoserkirchweg 63, 41747 Viersen,

E-Mail: kreth@akh-viersen.de

Stefan Osche, Deutsches Rotes Kreuz, Generalsekretariat, Carstennstraße 58, 12205 Berlin, E-Mail: osches@drk.de

Dr. med. Sebastian Russo, Zentrum Anaesthesiologie, Rettungs- und Intensivmedizin, Universitätsmedizin Göttingen, Robert-Koch-Str. 40, 37075 Göttingen, E-Mail: s.russo@medizin.uni-goettingen.de

Dr. med. Michael Sasse, Medizinische Hochschule Hannover, Pädiatrische Intensivmedizin, Carl-Neuberg-Str. 1, 30625 Hannover, E-Mail: sasse.michael@mh-hannover.de

Dr. med. Jens Chr. Schwindt, Univ. Klinik für Kinder- und Jugendheilkunde, Medizinische Universität Wien, Währinger Gürtel 18-20, A-1090 Wien, E-Mail: jens.schwindt@meduniwien.ac.at

Prof. Dr. med. Volker Wenzel, Universitätsklinik Innsbruck, Anichstraße 35, A-6020 Innsbruck, E-Mail: volker.wenzel@uki.at

\section{Interessenkonflikt}

Die Angaben zu möglichen Interessenkonflikten der Übersetzer finden Sie am Ende jeder Sektion.

Die Angaben zu möglichen Interessenskonflikten der Autoren der englischsprachigen

Originalpublikation finden Sie im Appendix B der Originalpublikation: Resuscitation 81(2010): 1261-1262.

Anzugeben waren: Finanzielle Verbindungen zu Unternehmen, Beschäftigungsverhältnisse, Beratungstätigkeiten, Aktienbesitz, Honorare für Vorträge, Reisekostenübernahmen, Studienunterstützungen oder andere Drittmittel, Verbindungen zu Organisationen, Krankenkassen, Politik oder sonstigen Interessengruppen, persönliche Interessenkonflikte (partnerschaftliche oder familiäre Verbindungen zu jemandem, dessen wirtschaftliche oder ideelle Belange durch den Beitrag berührt werden). 